\title{
Lyapunov-type inequalities for certain higher-order difference equations with mixed non-linearities
}

\section{Haidong Liu ${ }^{1 *}$}

\section{"Correspondence:}

tomlhd983@163.com

${ }^{1}$ School of Mathematical Sciences,

Qufu Normal University, Qufu,

P.R. China

\begin{abstract}
In this paper, we establish some new Lyapunov-type inequalities for some higher-order difference equations with boundary conditions. The obtained inequalities generalize the existing results in the literature.
\end{abstract}

Keywords: Lyapunov-type inequality; Difference equation; Higher-order; Anti-periodic boundary conditions

\section{Introduction}

During the past decades, continuous and discrete integral inequalities have attracted the attention of many researchers (see [1-59] and the references therein). Particularly, there have been plenty of references focused on the Lyapunov-type inequality and many of its generalizations due to its broad applications in the study of various properties of solutions of differential and difference equations such as oscillation theory, disconjugacy, and eigenvalue problems (see $[1,2,5-7,9,13,15,21,24,27-29,37,39,45,48,57,59]$ and the references therein).

Compared with a large number of references devoted to continuous Lyapunov-type inequalities, there is not much done for discrete Lyapunov-type inequalities (see $[6,13,21$, 29, 39, 59] and the references therein). For example, Zhang and Tang [29] considered the following even order difference equation:

$$
\triangle^{2 k} u(n)+(-1)^{k-1} q(n) u(n+1)=0,
$$

where $\Delta$ is the usual forward difference operator defined by $\Delta u(n)=u(n+1)-u(n), k \in \mathbb{N}$, $n \in \mathbb{Z}$ and $q(n)$ is a real-valued function defined on $\mathbb{Z}$. Under the following boundary conditions

$$
\triangle^{2 i} u(a)=\triangle^{2 i} u(b)=0, \quad i=0,1, \ldots, k-1 ; \quad u(n) \not \equiv 0, \quad n \in \mathbb{Z}[a, b],
$$

where $a, b \in \mathbb{N}, \mathbb{Z}[a, b]=\{a, a+1, \ldots, b-1, b\}$, they obtained the following result:

(c) The Author(s) 2018. This article is distributed under the terms of the Creative Commons Attribution 4.0 International License (http://creativecommons.org/licenses/by/4.0/), which permits unrestricted use, distribution, and reproduction in any medium, provided you give appropriate credit to the original author(s) and the source, provide a link to the Creative Commons license, and indicate if changes were made. 
Assume that $k \in \mathbb{N}$ and $q(n)$ is a real-valued function on $\mathbb{Z}$. If (1) has a solution $u(n)$ satisfying the boundary conditions (2), then

$$
\sum_{n=a}^{b-1}[|q(n)|(n-a+1)(b-n-1)] \geq \frac{2^{3(k-1)}}{(b-a)^{2 k-3}} .
$$

Recently, Liu and Tang [21] studied the following $m$-order difference equation:

$$
\left|\triangle^{m} u(n)\right|^{p-2} \triangle^{m} u(n)+r(n)|u(n)|^{p-2} u(n)=0,
$$

where $m \in \mathbb{N}, n \in \mathbb{Z}$ and $r(n)$ is a real-valued function defined on $\mathbb{Z}, p>1$ is a constant, and $u(n)$ satisfies the following anti-periodic boundary conditions:

$$
\triangle^{i} u(a)+\triangle^{i} u(b)=0, \quad i=0,1, \ldots, m-1 ; \quad u(n) \not \equiv 0, \quad n \in \mathbb{Z}[a, b],
$$

and they obtained the following result:

If (4) has a nonzero solution $u(n)$ satisfying the anti-periodic boundary conditions (5), then

$$
\sum_{n=a}^{b-1}|r(n)|^{q} \geq \frac{2^{m p}}{(b-a)^{m p-1}}
$$

where $q$ is a conjugate exponent of $p$.

In the present paper, we shall establish a new discrete Lyapunov-type inequality for the following $m$-order difference equation with mixed nonlinearities:

$$
\left|\triangle^{m} u(n)\right|^{p-2} \triangle^{m} u(n)+\sum_{i=0}^{m-1} r_{i}(n)\left|\triangle^{i} u(n)\right|^{p-2} \triangle^{i} u(n)=0,
$$

with the anti-periodic boundary conditions (5), where $m \in \mathbb{N}, n \in \mathbb{Z}, p>1$ is a constant and $r_{i}(n)(i=0,1, \ldots, m-1)$ are real-valued functions defined on $\mathbb{Z}$. Further, we will also prove a new Lyapunov-type inequality for the $2 m$-order difference equation

$$
\left|\Delta^{2 m} u(n)\right|^{p-2} \triangle^{2 m} u(n)+(-1)^{m-1} r(n)|u(n+1)|^{q-2} u(n+1)=0,
$$

with the following boundary conditions:

$$
\triangle^{2 i} u(a)=\triangle^{2 i} u(b)=0, \quad i=0,1, \ldots, m-1 ; \quad u(n) \not \equiv 0, \quad n \in \mathbb{Z}[a, b],
$$

where $m \in \mathbb{N}, p \geq q>2$ are constants, $n \in \mathbb{Z}$ and $r(n)$ is a real-valued function defined on $\mathbb{Z}$. Our works extend the results in [21] and [29].

\section{Main results}

Lemma 2.1 ([1]) If $A$ is positive and $B, z$ are nonnegative, then

$$
A z^{2 \tau}-B z^{\sigma}+\Gamma_{\sigma \tau} A^{-\sigma /(2-\sigma)} B^{2 \tau /(2-\sigma)} \geq 0
$$


for any $\sigma \in(0,2 \tau)$, where

$$
\Gamma_{\sigma \tau}=(2 \tau-\sigma) \sigma^{\sigma /(2 \tau-\sigma)} \tau^{-2 \tau /(2 \tau-\sigma)} 2^{-2 \tau /(2 \tau-\sigma)}>0
$$

with equality holding if and only if $B=z=0$.

Lemma $2.2([29])$ Assume that $u(n)$ is a real-valued function on $\mathbb{Z}[a, b], u(a)=u(b)=0$.

Then

$$
\begin{aligned}
& |u(n)| \leq \frac{(n-a)(b-n)}{b-a} \sum_{s=a}^{b-1}\left|\triangle^{2} u(s)\right|, \quad \forall n \in \mathbb{Z}(a, b-1), \\
& \sum_{n=a}^{b-1}|u(n)| \leq \frac{1}{2} \sum_{n=a}^{b-1}\left[(n-a+1)(b-n-1)\left|\Delta^{2} u(n)\right|\right] \leq \frac{(b-a)^{2}}{8} \sum_{n=a}^{b-1}\left|\Delta^{2} u(n)\right| .
\end{aligned}
$$

We now state the main theorem of this paper.

Theorem 2.1 If $u(n)$ is a nonzero solution of Eq. (7) satisfying the anti-periodic boundary conditions (5), then

$$
\sum_{i=0}^{m-1} \frac{(b-a)^{(m-i-1 / p)(p-1)}}{2^{(m-i)(p-1)}}\left(\sum_{n=a}^{b-1}\left|r_{i}(n)\right|^{q}\right)^{1 / q} \geq 1,
$$

where $q$ is the Hölder conjugate exponent of $p$, i.e., $1 / p+1 / q=1$.

Proof Since the nonzero solution $u(n)$ of Eq. (7) satisfies the anti-periodic boundary conditions (5), then $u(a)+u(b)=0$. For $n \in \mathbb{Z}[a, b]$, we have

$$
\begin{aligned}
u(n) & =u(n)-\frac{1}{2}[u(a)+u(b)]=\frac{1}{2} \sum_{k=a}^{n-1}[u(k+1)-u(k)]-\frac{1}{2} \sum_{k=n}^{b-1}[u(k+1)-u(k)] \\
& =\frac{1}{2} \sum_{k=a}^{n-1} \Delta u(k)-\frac{1}{2} \sum_{k=n}^{b-1} \Delta u(k) .
\end{aligned}
$$

Then

$$
|u(n)| \leq \frac{1}{2} \sum_{k=a}^{b-1}|\triangle u(k)| .
$$

Applying discrete Hölder's inequality

$$
\sum_{k=a}^{b-1}|f(k) g(k)| \leq\left(\sum_{k=a}^{b-1}|f(k)|^{\alpha}\right)^{1 / \alpha}\left(\sum_{k=a}^{b-1}|g(k)|^{\beta}\right)^{1 / \beta}
$$

to (15) with $f(k)=1, g(k)=|\Delta u(k)|, \alpha=q$, and $\beta=p$, we obtain that

$$
|u(n)| \leq \frac{1}{2} \sum_{k=a}^{b-1}|\Delta u(k)| \leq \frac{1}{2}(b-a)^{1 / q}\left(\sum_{k=a}^{b-1}|\Delta u(k)|^{p}\right)^{1 / p} .
$$


Liu Advances in Difference Equations ( 2018) 2018:229

Page 4 of 14

Similarly, we get

$$
\begin{aligned}
\left|\triangle^{i} u(n)\right| & \leq \frac{1}{2} \sum_{k=a}^{b-1}\left|\triangle^{i+1} u(k)\right| \\
& \leq \frac{1}{2}(b-a)^{1 / q}\left(\sum_{k=a}^{b-1}\left|\triangle^{i+1} u(k)\right|^{p}\right)^{1 / p}, \quad i=1,2, \ldots, m-1 .
\end{aligned}
$$

Then

$$
\left|\triangle^{i} u(n)\right|^{p} \leq\left(\frac{1}{2}\right)^{p}(b-a)^{p / q} \sum_{k=a}^{b-1}\left|\triangle^{i+1} u(k)\right|^{p}, \quad i=1,2, \ldots, m-1 .
$$

Summing (19) from $a$ to $b-1$, we have

$$
\sum_{n=a}^{b-1}\left|\Delta^{i} u(n)\right|^{p} \leq(b-a)\left(\frac{1}{2}\right)^{p}(b-a)^{p / q} \sum_{k=a}^{b-1}\left|\triangle^{i+1} u(k)\right|^{p}, \quad i=1,2, \ldots, m-1,
$$

i.e.,

$$
\left(\sum_{n=a}^{b-1}\left|\triangle^{i} u(n)\right|^{p}\right)^{1 / p} \leq \frac{b-a}{2}\left(\sum_{k=a}^{b-1}\left|\triangle^{i+1} u(k)\right|^{p}\right)^{1 / p}, \quad i=1,2, \ldots, m-1 .
$$

From (21), we obtain

$$
\begin{aligned}
& \left(\sum_{n=a}^{b-1}\left|\triangle^{i} u(n)\right|^{p}\right)^{1 / p} \\
& \quad \leq \frac{b-a}{2}\left(\sum_{k=a}^{b-1}\left|\triangle^{i+1} u(k)\right|^{p}\right)^{1 / p} \\
& \quad \leq\left(\frac{b-a}{2}\right)^{2}\left(\sum_{k=a}^{b-1}\left|\triangle^{i+2} u(k)\right|^{p}\right)^{1 / p} \\
& \quad \leq \ldots \\
& \quad \leq\left(\frac{b-a}{2}\right)^{m-i}\left(\sum_{k=a}^{b-1}\left|\triangle^{m} u(k)\right|^{p}\right)^{1 / p}, \quad i=1,2, \ldots, m-1 .
\end{aligned}
$$

Then, from (17) and (22) for $i=1$, we obtain

$$
|u(n)| \leq \frac{1}{2}(b-a)^{1 / q}\left(\frac{b-a}{2}\right)^{m-1}\left(\sum_{k=a}^{b-1}\left|\Delta^{m} u(k)\right|^{p}\right)^{1 / p}
$$

and by (18) and (22), we get

$$
\left|\triangle^{i} u(n)\right| \leq \frac{1}{2}(b-a)^{1 / q}\left(\frac{b-a}{2}\right)^{m-i-1}\left(\sum_{k=a}^{b-1}\left|\Delta^{m} u(k)\right|^{p}\right)^{1 / p}, \quad i=1,2, \ldots, m-1
$$


Multiplying (7) by $\triangle^{m} u(n)$, we have

$$
\left|\triangle^{m} u(n)\right|^{p}+\sum_{i=0}^{m-1} r_{i}(n)\left|\triangle^{i} u(n)\right|^{p-2} \triangle^{i} u(n) \triangle^{m} u(n)=0 .
$$

Then we get

$$
\begin{aligned}
\left|\triangle^{m} u(n)\right|^{p} & =-\sum_{i=0}^{m-1} r_{i}(n)\left|\triangle^{i} u(n)\right|^{p-2} \triangle^{i} u(n) \triangle^{m} u(n) \\
& \leq \sum_{i=0}^{m-1}\left|r_{i}(n)\right|\left|\triangle^{i} u(n)\right|^{p-2}\left|\triangle^{i} u(n)\right|\left|\triangle^{m} u(n)\right| \\
& =\sum_{i=0}^{m-1}\left|r_{i}(n)\right|\left|\triangle^{i} u(n)\right|^{p-1}\left|\triangle^{m} u(n)\right| .
\end{aligned}
$$

Summing (26) from $a$ to $b-1$, we have

$$
\begin{aligned}
\sum_{n=a}^{b-1}\left|\triangle^{m} u(n)\right|^{p} \leq & \sum_{i=0}^{m-1} \sum_{n=a}^{b-1}\left|r_{i}(n)\right|\left|\triangle^{i} u(n)\right|^{p-1}\left|\triangle^{m} u(n)\right| \\
= & \sum_{n=a}^{b-1}\left|r_{0}(n)\right||u(n)|^{p-1}\left|\triangle^{m} u(n)\right| \\
& +\sum_{i=1}^{m-1} \sum_{n=a}^{b-1}\left|r_{i}(n)\right|\left|\triangle^{i} u(n)\right|^{p-1}\left|\triangle^{m} u(n)\right| .
\end{aligned}
$$

For the first summation on the right-hand side of (27), from (23) and Hölder's inequality (16), we obtain that

$$
\begin{aligned}
\sum_{n=a}^{b-1} \mid & \left.r_{0}(n)|| u(n)\right|^{p-1}\left|\Delta^{m} u(n)\right| \\
\leq & \left(\frac{1}{2}\right)^{p-1}(b-a)^{(p-1) / q}\left(\frac{b-a}{2}\right)^{(m-1)(p-1)} \\
& \cdot\left(\sum_{n=a}^{b-1}\left|\Delta^{m} u(n)\right|^{p}\right)^{(p-1) / p} \sum_{n=a}^{b-1}\left|r_{0}(n)\right|\left|\Delta^{m} u(n)\right| \\
= & \frac{(b-a)^{(m-1 / p)(p-1)}}{2^{m(p-1)}}\left(\sum_{n=a}^{b-1}\left|\Delta^{m} u(n)\right|^{p}\right)^{(p-1) / p} \sum_{n-1}^{b-1}\left|r_{0}(n)\right|\left|\Delta^{m} u(n)\right| \\
\leq & \frac{(b-a)^{(m-1 / p)(p-1)}}{2^{m(p-1)}}\left(\sum_{n=a}^{b-1}\left|\Delta^{m} u(n)\right|^{p}\right)^{(p-1) / p} \\
& \cdot\left(\sum_{n=a}^{b-1}\left|r_{0}(n)\right|^{q}\right)^{1 / q}\left(\sum_{n=a}^{b-1}\left|\Delta^{m} u(n)\right|^{p}\right)^{1 / p} \\
= & \frac{(b-a)^{(m-1 / p)(p-1)}}{2^{m(p-1)}}\left(\sum_{n=a}^{b-1}\left|\Delta^{m} u(n)\right|^{p}\right)^{\left(\sum_{n=a}^{b-1}\left|r_{0}(n)\right|^{q}\right)^{1 / q} \cdot}
\end{aligned}
$$


On the other hand, for the second summation on the right-hand side of (27), from (24) and Hölder's inequality (16), we have that

$$
\begin{aligned}
\sum_{n=a}^{b-1} \mid & \left.r_{i}(n)|| \Delta^{i} u(n)\right|^{p-1}\left|\Delta^{m} u(n)\right| \\
\leq & \left(\frac{1}{2}\right)^{p-1}(b-a)^{(p-1) / q}\left(\frac{b-a}{2}\right)^{(m-i-1)(p-1)} \\
& \cdot\left(\sum_{n=a}^{b-1}\left|\Delta^{m} u(n)\right|^{p}\right)^{(p-1) / p} \sum_{n=a}^{b-1}\left|r_{i}(n)\right|\left|\Delta^{m} u(n)\right| \\
= & \frac{(b-a)^{(m-i-1 / p)(p-1)}}{2^{(m-i)(p-1)}}\left(\sum_{n=a}^{b-1}\left|\Delta^{m} u(n)\right|^{p}\right)^{(p-1) / p} \sum_{n=a}^{b-1}\left|r_{i}(n)\right|\left|\Delta^{m} u(n)\right| \\
\leq & \frac{(b-a)^{(m-i-1 / p)(p-1)}}{2^{(m-i)(p-1)}}\left(\sum_{n=a}^{b-1}\left|\Delta^{m} u(n)\right|^{p}\right)^{(p-1) / p} \\
& \cdot\left(\sum_{n=a}^{b-1}\left|r_{i}(n)\right|^{q}\right)^{1 / q}\left(\sum_{n=a}^{b-1}\left|\Delta^{m} u(n)\right|^{p}\right)^{1 / p} \\
= & \frac{(b-a)^{(m-i-1 / p)(p-1)}}{2^{(m-i)(p-1)}}\left(\sum_{n=a}^{b-1}\left|\Delta^{m} u(n)\right|^{p}\right)\left(\sum_{n=a}^{b-1}\left|r_{i}(n)\right|^{q}\right)^{1 / q}, \quad i=1,2, \ldots, m-1,
\end{aligned}
$$

and then

$$
\begin{aligned}
& \sum_{i=1}^{m-1} \sum_{n=a}^{b-1}\left|r_{i}(n)\right|\left|\Delta^{i} u(n)\right|^{p-1}\left|\Delta^{m} u(n)\right| \\
& \quad \leq \sum_{i=1}^{m-1} \frac{(b-a)^{(m-i-1 / p)(p-1)}}{2^{(m-i)(p-1)}}\left(\sum_{n=a}^{b-1}\left|\Delta^{m} u(n)\right|^{p}\right)\left(\sum_{n=a}^{b-1}\left|r_{i}(n)\right|^{q}\right)^{1 / q} \\
& \quad=\left(\sum_{n=a}^{b-1}\left|\Delta^{m} u(n)\right|^{p}\right) \sum_{i=1}^{m-1} \frac{(b-a)^{(m-i-1 / p)(p-1)}}{2^{(m-i)(p-1)}}\left(\sum_{n=a}^{b-1}\left|r_{i}(n)\right|^{q}\right)^{1 / q} .
\end{aligned}
$$

By (27), (28), and (30), we get

$$
\begin{aligned}
\sum_{n=a}^{b-1}\left|\Delta^{m} u(n)\right|^{p} & \\
\leq & \frac{(b-a)^{(m-1 / p)(p-1)}}{2^{m(p-1)}}\left(\sum_{n=a}^{b-1}\left|\Delta^{m} u(n)\right|^{p}\right)\left(\sum_{n=a}^{b-1}\left|r_{0}(n)\right|^{q}\right)^{1 / q} \\
& +\left(\sum_{n=a}^{b-1}\left|\Delta^{m} u(n)\right|^{p}\right) \sum_{i=1}^{m-1} \frac{(b-a)^{(m-i-1 / p)(p-1)}}{2^{(m-i)(p-1)}}\left(\sum_{n=a}^{b-1}\left|r_{i}(n)\right|^{q}\right)^{1 / q} .
\end{aligned}
$$

Now, we claim that $\sum_{n=a}^{b-1}|\Delta u(n)|^{p}>0$. In fact, if the above inequality is not true, we have $\sum_{n=a}^{b-1}|\Delta u(n)|^{p}=0$, then $\Delta u(n)=0$ for $n \in \mathbb{Z}[a, b-1]$. By the anti-periodic conditions (5), we obtain $u(n)=0$ for $n \in \mathbb{Z}[a, b]$, which contradicts $u(n) \not \equiv 0, n \in \mathbb{Z}[a, b]$. From (22), we 
get $\sum_{n=a}^{b-1}\left|\triangle^{m} u(n)\right|^{p}>0$. Thus, dividing both sides of (31) by $\sum_{n=a}^{b-1}\left|\triangle^{m} u(n)\right|^{p}$, we obtain

$$
\begin{aligned}
1 \leq & \frac{(b-a)^{(m-1 / p)(p-1)}}{2^{m(p-1)}}\left(\sum_{n=a}^{b-1}\left|r_{0}(n)\right|^{q}\right)^{1 / q} \\
& +\sum_{i=1}^{m-1} \frac{(b-a)^{(m-i-1 / p)(p-1)}}{2^{(m-i)(p-1)}}\left(\sum_{n=a}^{b-1}\left|r_{i}(n)\right|^{q}\right)^{1 / q} \\
= & \sum_{i=0}^{m-1} \frac{(b-a)^{(m-i-1 / p)(p-1)}}{2^{(m-i)(p-1)}}\left(\sum_{n=a}^{b-1}\left|r_{i}(n)\right|^{q}\right)^{1 / q} .
\end{aligned}
$$

This completes the proof of Theorem 2.1.

Remark If $r_{i}(n) \equiv 0, i=1,2, \ldots, m-1$, then Theorem 2.1 coincides with Theorem 1 in [21].

Let $p=2, m=2 k, k \in \mathbb{N}$ in Theorem 2.1 , we have the following corollary.

Corollary 2.1 If $u(n)$ is a nonzero solution of

$$
\triangle^{2 k} u(n)+\sum_{i=0}^{2 k-1} r_{i}(n) \triangle^{i} u(n)=0
$$

and satisfies the anti-periodic boundary conditions

$$
\triangle^{i} u(a)+\triangle^{i} u(b)=0, \quad i=0,1, \ldots, 2 k-1 ; \quad u(n) \not \equiv 0, \quad n \in \mathbb{Z}[a, b]
$$

then

$$
\sum_{i=0}^{2 k-1} \frac{(b-a)^{2 k-i-1 / 2}}{2^{2 k-i}}\left(\sum_{n=a}^{b-1}\left|r_{i}(n)\right|^{2}\right)^{1 / 2} \geq 1 .
$$

Let $p=2, m=2 k-1, k \in \mathbb{N}$ in Theorem 2.1, we have the following corollary.

Corollary 2.2 If $u(n)$ is a nonzero solution of

$$
\triangle^{2 k-1} u(n)+\sum_{i=0}^{2 k-2} r_{i}(n) \triangle^{i} u(n)=0
$$

and satisfies the anti-periodic boundary conditions

$$
\triangle^{i} u(a)+\triangle^{i} u(b)=0, \quad i=0,1, \ldots, 2 k-2 ; \quad u(n) \not \equiv 0, \quad n \in \mathbb{Z}[a, b]
$$

then

$$
\sum_{i=0}^{2 k-2} \frac{(b-a)^{2 k-i-3 / 2}}{2^{2 k-1-i}}\left(\sum_{n=a}^{b-1}\left|r_{i}(n)\right|^{2}\right)^{1 / 2} \geq 1 .
$$

Let $m=2$ in Theorem 2.1, we have the following corollary. 
Corollary 2.3 If $u(n)$ is a nonzero solution of

$$
\left|\triangle^{2} u(n)\right|^{p-2} \triangle^{2} u(n)+\sum_{i=0}^{1} r_{i}(n)\left|\triangle^{i} u(n)\right|^{p-2} \triangle^{i} u(n)=0
$$

and satisfies the anti-periodic boundary conditions

$$
\triangle^{i} u(a)+\triangle^{i} u(b)=0, \quad i=0,1 ; \quad u(n) \not \equiv 0, \quad n \in \mathbb{Z}[a, b],
$$

then

$$
\sum_{i=0}^{1} \frac{(b-a)^{(2-i-1 / p)(p-1)}}{2^{(2-i)(p-1)}}\left(\sum_{n=a}^{b-1}\left|r_{i}(n)\right|^{q}\right)^{1 / q} \geq 1 .
$$

Next, we establish a Lyapunov-type inequality for Eq. (8).

Theorem 2.2 If $u(n)$ is a nonzero solution of Eq. (8) satisfying the anti-periodic boundary conditions (9), then

$$
\left(\sum_{n=a}^{b-1}|r(n)|\right)^{1 /(p-1)}>\frac{2^{3 m-2}}{(b-a)^{2 m-1 /(p-1)}} \frac{1}{\sqrt{\Gamma_{\frac{q-1}{p-1} 1}}},
$$

where

$$
\Gamma_{\frac{q-1}{p-1} 1}=\left(\frac{2 p-q-1}{p-1}\right)\left(\frac{q-1}{p-1}\right)^{(q-1) /(2 p-q-1)} 2^{2(1-p) /(2 p-q-1)} .
$$

Proof Choose $c \in \mathbb{Z}[a, b]$ such that $|u(c)|=\max _{n \in \mathbb{Z}[a, b]}|u(n)|$. Since (9), it follows from Lemma 2.2 that

$$
|u(c)| \leq \frac{(c-a)(b-c)}{b-a} \sum_{n=a}^{b-1}\left|\triangle^{2} u(n)\right| \leq \frac{b-a}{4} \sum_{n=a}^{b-1}\left|\Delta^{2} u(n)\right|
$$

and

$$
\sum_{n=a}^{b-1}\left|\Delta^{2 i} u(n)\right| \leq \frac{(b-a)^{2}}{8} \sum_{n=a}^{b-1}\left|\Delta^{2 i+2} u(n)\right|, \quad i=1,2, \ldots, m-1 .
$$

From (40) and (41), we obtain

$$
\begin{aligned}
|u(c)| & \leq \frac{b-a}{4} \sum_{n=a}^{b-1}\left|\triangle^{2} u(n)\right| \\
& \leq \frac{b-a}{4} \frac{(b-a)^{2}}{8} \sum_{n=a}^{b-1}\left|\Delta^{4} u(n)\right| \\
& \leq \frac{b-a}{4}\left(\frac{(b-a)^{2}}{8}\right)^{2} \sum_{n=a}^{b-1}\left|\Delta^{6} u(n)\right|
\end{aligned}
$$




$$
\begin{aligned}
& \leq \cdots \\
& \leq \frac{b-a}{4}\left(\frac{(b-a)^{2}}{8}\right)^{m-1} \sum_{n=a}^{b-1}\left|\Delta^{2 m} u(n)\right| .
\end{aligned}
$$

Applying discrete Hölder's inequality (16) to the summation on the right-hand side of (42) with $f(n)=1, g(n)=\left|\triangle^{2 m} u(n)\right|, \alpha=\frac{p-1}{p-2}$, and $\beta=p-1$, we obtain that

$$
\begin{aligned}
|u(c)| & \leq \frac{b-a}{4}\left(\frac{(b-a)^{2}}{8}\right)^{m-1}(b-a)^{(p-2) /(p-1)}\left(\sum_{k=a}^{b-1}\left|\triangle^{2 m} u(n)\right|^{p-1}\right)^{1 /(p-1)} \\
& =\frac{(b-a)^{2 m-1 /(p-1)}}{2^{3 m-1}}\left(\sum_{k=a}^{b-1}\left|\triangle^{2 m} u(n)\right|^{p-1}\right)^{1 /(p-1)} \cdot
\end{aligned}
$$

On the other hand, from (8), we have

$$
\left|\triangle^{2 m} u(n)\right|^{p-2} \triangle^{2 m} u(n)=(-1)^{m} r(n)|u(n+1)|^{q-2} u(n+1),
$$

then

$$
\left|\triangle^{2 m} u(n)\right|^{p-1}=|r(n)||u(n+1)|^{q-1} .
$$

Summing (45) from $a$ to $b-1$, we have

$$
\sum_{n=a}^{b-1}\left|\Delta^{2 m} u(n)\right|^{p-1}=\sum_{n=a}^{b-1}|r(n)||u(n+1)|^{q-1}
$$

then

$$
\left(\sum_{n=a}^{b-1}\left|\triangle^{2 m} u(n)\right|^{p-1}\right)^{1 /(p-1)}=\left(\sum_{n=a}^{b-1}|r(n)||u(n+1)|^{q-1}\right)^{1 /(p-1)}
$$

From (43) and (47), we have

$$
\begin{aligned}
|u(c)| & \leq \frac{(b-a)^{2 m-1 /(p-1)}}{2^{3 m-1}}\left(\sum_{n=a}^{b-1}|r(n)||u(n+1)|^{q-1}\right)^{1 /(p-1)} \\
& \leq \frac{(b-a)^{2 m-1 /(p-1)}}{2^{3 m-1}}\left(\sum_{n=a}^{b-1}|r(n)|\right)^{1 /(p-1)}|u(c)|^{(q-1) /(p-1)} \\
& \leq \mathcal{K}|u(c)|^{(q-1) /(p-1)}
\end{aligned}
$$

where

$$
\mathcal{K}=\frac{(b-a)^{2 m-1 /(p-1)}}{2^{3 m-1}}\left(\sum_{n=a}^{b-1}|r(n)|\right)^{1 /(p-1)}
$$


Using inequality (10) in Lemma 2.1 with $A=B=1, z=|u(c)|, \tau=1, \sigma=\frac{q-1}{p-1}$, we have

$$
|u(c)|^{2}-|u(c)|^{(q-1) /(p-1)}+\Gamma_{\frac{q-1}{p-1} 1}>0 .
$$

From (48) and (50), we get

$$
|u(c)|^{2}-\frac{1}{\mathcal{K}}|u(c)|+\Gamma_{\frac{q-1}{p-1} 1}>0 .
$$

This is possible only if

$$
\frac{1}{\mathcal{K}^{2}}-4 \Gamma_{\frac{q-1}{p-1} 1}<0
$$

i.e.,

$$
\mathcal{K}>\frac{1}{2 \sqrt{\Gamma \frac{q-1}{p-1} 1}}
$$

From (49) and (53), we obtain

$$
\frac{(b-a)^{2 m-1 /(p-1)}}{2^{3 m-1}}\left(\sum_{n=a}^{b-1}|r(n)|\right)^{1 /(p-1)}>\frac{1}{2 \sqrt{\Gamma_{\frac{q-1}{p-1}} 1}} .
$$

Thus, (38) holds. This completes the proof of Theorem 2.2.

For $p>q=2$, using a method similar to Theorem 2.2, we have the following theorem.

Theorem 2.3 If $u(n)$ is a nonzero solution of

$$
\left|\triangle^{2 m} u(n)\right|^{p-2} \triangle^{2 m} u(n)+(-1)^{m-1} r(n) u(n+1)=0,
$$

satisfying the anti-periodic boundary conditions (9), then

$$
\left(\sum_{n=a}^{b-1}|r(n)|\right)^{1 /(p-1)}>\frac{2^{3 m-2}}{(b-a)^{2 m-1 /(p-1)}} \frac{1}{\sqrt{\Gamma_{\frac{1}{p-1}}}}
$$

where

$$
\Gamma_{\frac{1}{p-1} 1}=\left(\frac{2 p-3}{p-1}\right)\left(\frac{1}{p-1}\right)^{1 /(2 p-3)} 2^{2(1-p) /(2 p-3)} .
$$

Remark For $p=q=2$, using a method similar to Theorem 2.2, we have that the result coincides with Corollary 2.3 in [29].

Let $m=1$ in Theorem 2.2, we have the following corollary. 
Corollary 2.4 If $u(n)$ is a nonzero solution of

$$
\left|\triangle^{2} u(n)\right|^{p-2} \triangle^{2} u(n)+r(n)|u(n+1)|^{q-2} u(n+1)=0
$$

and satisfies the anti-periodic boundary conditions

$$
u(a)=u(b)=0, \quad u(n) \not \equiv 0, \quad n \in \mathbb{Z}[a, b],
$$

then

$$
\left(\sum_{n=a}^{b-1}|r(n)|\right)^{1 /(p-1)}>\frac{2}{(b-a)^{2-1 /(p-1)}} \frac{1}{\sqrt{\Gamma_{\frac{q-1}{p-1} 1}}},
$$

where $\Gamma_{\frac{q-1}{p-1} 1}$ is defined as in (39).

Let $m=1$ in Theorem 2.3, we have the following corollary.

Corollary 2.5 If $u(n)$ is a nonzero solution of

$$
\left|\triangle^{2} u(n)\right|^{p-2} \triangle^{2} u(n)+r(n) u(n+1)=0
$$

and satisfies the anti-periodic boundary conditions

$$
u(a)=u(b)=0, \quad u(n) \not \equiv 0, \quad n \in \mathbb{Z}[a, b],
$$

then

$$
\left(\sum_{n=a}^{b-1}|r(n)|\right)^{1 /(p-1)}>\frac{2}{(b-a)^{2-1 /(p-1)}} \frac{1}{\sqrt{\Gamma_{\frac{1}{p-1}}}},
$$

where $\Gamma_{\frac{1}{p-1}}$ is defined as in (57).

\section{Applications}

In this section, we investigate the nonexistence and uniqueness for solutions of certain BVPs. First, we consider the nonexistence for solutions of the BVP consisting of (7) and the boundary conditions (5).

Theorem 3.1 Assume

$$
\sum_{i=0}^{m-1} \frac{(b-a)^{(m-i-1 / p)(p-1)}}{2^{(m-i)(p-1)}}\left(\sum_{n=a}^{b-1}\left|r_{i}(n)\right|^{q}\right)^{1 / q}<1,
$$

where $q$ is the Hölder conjugate exponent of $p$, i.e., $1 / p+1 / q=1$. Then BVP (7), (5) has no nontrivial solution. 
Proof Assume the contrary. Then BVP (7), (5) has a nontrivial solution $u(n)$. By Theorem 2.1, inequality (13) holds. This contradicts assumption (62).

Next, we consider the uniqueness for solutions of nonhomogeneous BVP consisting of the equation

$$
\triangle^{2 k} u(n)+\sum_{i=0}^{2 k-1} r_{i}(n) \triangle^{i} u(n)=f(n), \quad n \in \mathbb{Z}[A, B]
$$

and the boundary conditions

$$
\triangle^{i} u(a)+\triangle^{i} u(b)=M_{i}, \quad i=0,1, \ldots, 2 k-1 ; \quad n \in \mathbb{Z}[a, b],
$$

where $k \in \mathbb{N}, n \in \mathbb{Z}$, and $f, r_{i}(n)(i=0,1, \ldots, 2 k-1)$ are real-valued functions defined on $\mathbb{Z}$, $A, B, a, b \in \mathbb{N}, A<a<b<B$, and $M_{i} \in \mathbb{R}, i=0,1, \ldots, 2 k-1$.

\section{Theorem 3.2 Assume}

$$
\sum_{i=0}^{2 k-1} \frac{(B-A)^{(2 k-i-1 / 2)}}{2^{(2 k-i)}}\left(\sum_{n=A}^{B-1}\left|r_{i}(n)\right|^{2}\right)^{1 / 2}<1 .
$$

Then $B V P$ (63), (64) has at most one solution on $(A, B)$ for any $a, b \in(A, B), M_{i} \in \mathbb{R}$, $i=0,1, \ldots, 2 k-1$.

Proof Let $u_{1}(n)$ and $u_{2}(n)$ be two solutions of BVP (63), (64) in $(A, B)$. Define $u(n)=$ $u_{1}(n)-u_{2}(n)$. Then $u(n)$ is a solution of BVP (32), (33). Then, by Theorem 3.1 with $p=2$ and $m=2 k$, we have $u(n) \equiv 0$, i.e., $u_{1}(n) \equiv u_{2}(n)$. This shows that BVP (63), (64) has at most one solution on $(A, B)$.

\section{Acknowledgements}

The author is indebted to the anonymous referees for their valuable suggestions and helpful comments which helped improve the paper significantly.

\section{Funding}

This research was supported by the Natural Science Foundation of Shandong Province (China) (Grant No. ZR2018MA018), A Project of Shandong Province Higher Educational Science and Technology Program (China) (Grant No. J14LI09), and the National Natural Science Foundation of China (Grant No. 11671227).

\section{Competing interests}

The author declares that there is no conflict of interests regarding the publication of this paper.

\section{Authors' contributions}

HDL organized and wrote this paper. Further, he examined all the steps of the proofs in this research. The author read and approved the final manuscript.

\section{Publisher's Note}

Springer Nature remains neutral with regard to jurisdictional claims in published maps and institutional affiliations.

Received: 2 May 2018 Accepted: 26 June 2018 Published online: 03 July 2018

\section{References}

1. Agarwal, R.P., Özbekler, A.: Disconjugacy via Lyapunov and Vallée-Poussin type inequalities for forced differential equations. Appl. Math. Comput. 265, 456-468 (2015)

2. Agarwal, R.P., Özbekler, A.: Lyapunov type inequalities for even order differential equations with mixed nonlinearities. J. Inequal. Appl. 2015, Article ID 142 (2015) 
3. Feng, Q.H., Meng, F.W., Zhang, Y.M., Zheng, B., Zhou, J.C.: Some nonlinear delay integral inequalities on time scales arising in the theory of dynamics equations. J. Inequal. Appl. 2011, Article ID 29 (2011)

4. Feng, Q.H., Meng, F.W., Zhang, Y.M.: Generalized Gronwall-Bellman-type discrete inequalities and their applications. J. Inequal. Appl. 2011, Article ID 47 (2011)

5. Çakmak, D.: Lyapunov-type integral inequalities for certain higher order differential equations. Appl. Math. Comput. 216, 368-373 (2010)

6. Cheng, S.S.: Lyapunov inequalities for differential and difference equations. Fasc. Math. 23, 25-41 (1991)

7. Dhar, S., Kong, Q.K.: Lyapunov-type inequalities for $\alpha$-th order fractional differential equations with $2<\alpha \leq 3$ and fractional boundary conditions. Electron. J. Differ. Equ. 2017, Article ID 203 (2017)

8. Du, L.W., Xu, R.: Some new Pachpatte type inequalities on time scales and their applications. J. Math. Inequal. 6(2), 229-240 (2012)

9. Eliason, S.B.: Lyapunov inequalities and bounds on solutions of certain second order equations. Can. Math. Bull. 17(4), 499-504 (1974)

10. Feng, Q.H., Meng, F.W., Zhang, Y.M.: Some new finite difference inequalities arising in the theory of difference equations. Adv. Differ. Equ. 2011, Article ID 21 (2011)

11. Feng, Q.H., Meng, F.W.: Some generalized Ostrowski-Gruss type integral inequalities. Comput. Math. Appl. 63(3), 652-659 (2012)

12. Gu, J., Meng, F.W.: Some new nonlinear Volterra-Fredholm type dynamic integral inequalities on time scales. Appl. Math. Comput. 245, 235-242 (2014)

13. Guseinov, G.S., Kaymakcalan, B.: Lyapunov inequalities for discrete linear Hamiltonian systems. Comput. Math. Appl. 45, 1399-1416 (2003)

14. Liu, H.D., Meng,F.W., Liu, P.C.: Oscillation and asymptotic analysis on a new generalized Emden-Fowler equation. Appl. Math. Comput. 219(5), 2739-2748 (2012)

15. Lee, C., Yeh, C., Hong, C., Agarwal, R.P.: Lyapunov and Wirtinger inequalities. Appl. Math. Lett. 17, 847-853 (2004)

16. Li, L.Z., Meng, F.W., He, L.L.: Some generalized integral inequalities and their applications. J. Math. Anal. Appl. 372(1), 339-349 (2010)

17. Liu, H.D., Meng, F.W.: Oscillation criteria for second order linear matrix differential systems with damping. J. Comput. Appl. Math. 229(1), 222-229 (2009)

18. Li, L.Z., Meng, F.W., Ju, P.J.: Some new integral inequalities and their applications in studying the stability of nonlinear integro-differential equations with time delay. J. Math. Anal. Appl. 377(2), 853-862 (2011)

19. Feng, Q.H., Meng, F.W.: Generalized Ostrowski type inequalities for multiple points on time scales involving functions of two independent variables. J. Inequal. Appl., 2012, Article ID 74 (2012)

20. Liu, H.D.: Some new integral inequalities with mixed nonlinearities for discontinuous functions. Adv. Differ. Equ. 2018 Article ID 22 (2018)

21. Liu, X.G., Tang, M.L.: Lyapunov-type inequality for higher order difference equations. Appl. Math. Comput. 232, 666-669 (2014)

22. Liu, H.D., Meng, F.W.: Some new nonlinear integral inequalities with weakly singular kernel and their applications to FDEs. J. Inequal. Appl. 2015, Article ID 209 (2015)

23. Feng, Q.H., Meng, F.W., Zheng, B.: Gronwall-Bellman type nonlinear delay integral inequalities on time scales. J. Math. Anal. Appl. 382(2), 772-784 (2011)

24. Lyapunov, A.M.: Probleme général de la stabilité du mouvement. Ann. Fac. Sci. Univ. Toulouse 2, 27-247 (1907) (French translation of a Russian paper dated 1893); reprinted as Ann. Math. Stud. 17 (1947)

25. Meng, F.W., Shao, J.: Some new Volterra-Fredholm type dynamic integral inequalities on time scales. Appl. Math. Comput. 223, 444-451 (2013)

26. Liu, H.D., Ma, C.Q.: Oscillation criteria for second-order neutral delay dynamic equations with nonlinearities given by Riemann-Stieltjes integrals. Abstr. Appl. Anal. 2013, Article ID 530457 (2013)

27. O'Regan, D., Samet, B.: Lyapunov-type inequalities for a class of fractional differential equations. J. Inequal. Appl. 2015, Article ID 247 (2015)

28. Tang, X.-H., Zhang, M.: Lyapunov inequalities and stability for linear Hamiltonian systems. J. Differ. Equ. 252, 358-381 (2012)

29. Zhang, Q.M., Tang, X.H.: Lyapunov-type inequalities for even order difference equations. Appl. Math. Lett. 25, 1830-1834 (2012)

30. Liu, H.D., Meng, F.W.: Nonlinear retarded integral inequalities on time scales and their applications. J. Math. Inequal. $12(1), 219-234(2018)$

31. Feng, Q.H., Meng, F.W., Fu, B.S.: Some new generalized Volterra-Fredholm type finite difference inequalities involving four iterated sums. Appl. Math. Comput. 219(15), 8247-8258 (2013)

32. Liu, H.D., Meng, F.W.: Some new generalized Volterra-Fredholm type discrete fractional sum inequalities and their applications. J. Inequal. Appl. 2016, Article ID 213 (2016)

33. Xu, R., Ma, X.T.: Some new retarded nonlinear Volterra-Fredholm type integral inequalities with maxima in two variables and their applications. J. Inequal. Appl. 2017, Article ID 187 (2017)

34. Xu, R., Zhang, Y.: Generalized Gronwall fractional summation inequalities and their applications. J. Inequal. Appl. 2015, Article ID 242 (2015)

35. Liu, H.D.: A class of retarded Volterra-Fredholm type integral inequalities on time scales and their applications. J. Inequal. Appl. 2017, Article ID 293 (2017)

36. Tian, Y.Z., Fan, M., Meng, F.W.: A generalization of retarded integral inequalities in two independent variables and their applications. Appl. Math. Comput. 221, 239-248 (2013)

37. Tiryaki, A., Çakmak, D., Aktas, M.F.: Lyapunov-type inequalities for a certain class of nonlinear systems. Comput. Math Appl. 64, 1804-1811 (2012)

38. Liu, H.D., Ma, C.Q.: Oscillation criteria of even order delay dynamic equations with nonlinearities given by Riemann-Stieltjes integrals. Abstr. Appl. Anal. 2014, Article ID 395381 (2014)

39. Unal, M., Çakmak, D., Tiryaki, A.: A discrete analogue of Lyapunov-type inequalities for nonlinear systems. Comput. Math. Appl. 55, 2631-2642 (2008) 
40. Wang, J.F., Meng, F.W., Gu, J.: Estimates on some power nonlinear Volterra-Fredholm type dynamic integral inequalities on time scales. Adv. Differ. Equ. 2017, Article ID 257 (2017)

41. Wang, T.L., Xu, R.: Some integral inequalities in two independent variables on time scales. J. Math. Inequal. 6(1), 107-118 (2012)

42. Liu, H.D., Liu, P.C.: Oscillation and asymptotic analysis on a new generalized Emden-Fowler equation. Abstr. Appl. Anal. 2013, Article ID 962590 (2013)

43. Wang, T.L., Xu, R.: Bounds for some new integral inequalities with delay on time scales. J. Math. Inequal. 6(3), 355-366 (2012)

44. Liu, H.D., Meng, F.W.: Interval oscillation criteria for second-order nonlinear forced differential equations involving variable exponent. Adv. Differ. Equ. 2016, Article ID 291 (2016)

45. Watanabe, K., Yamagishi, H., Kametaka, Y.: Riemann zeta function and Lyapunov-type inequalities for certain higher order differential equations. Appl. Math. Comput. 218, 3950-3953 (2011)

46. Xu, R., Meng, F.W., Song, C.H.: On some integral inequalities on time scales and their applications. J. Inequal. Appl. 2010, Article ID 464976 (2010)

47. Tunç, E., Liu, H.D.: Oscillatory behavior for second-order damped differential equation with nonlinearities including Riemann-Stieltjes integrals. Electron. J. Differ. Equ. 2018, Article ID 54 (2018)

48. Yang, X.J., Lo, K.M.: Lyapunov-type inequalities for a class of higher-order linear differential equations with anti-periodic boundary conditions. Appl. Math. Lett. 34, 33-36 (2014)

49. Yuan, Z.L., Yuan, X.W., Meng, F.W., Zhang, H.X.: Some new delay integral inequalities and their applications. Appl. Math. Comput. 208, 231-237 (2009)

50. Bai, Y.Z.: Backward solutions to nonlinear integro-differential systems. Cent. Eur. J. Math. 8(4), 807-815 (2010)

51. Xu, R.: Some new nonlinear weakly singular integral inequalities and their applications. J. Math. Inequal. 11(4), 1007-1018 (2017)

52. Liu, L.H., Bai, Y.Z.: New oscillation criteria for second-order nonlinear neutral delay differential equations. J. Comput. Appl. Math. 231(2), 657-663 (2009)

53. Bai, Y.Z., Zhang, P.P.: On a class of Volterra nonlinear equations of parabolic type. Appl. Math. Comput. 216(1), 236-240 (2010)

54. Zheng, Z.W.: Invariance of deficiency indices under perturbation for discrete Hamiltonian systems. J. Differ. Equ. Appl. $19(8), 1243-1250(2013)$

55. Zheng, Z.W., Wang, X., Han, H.M.: Oscillation criteria for forced second order differential equations with mixed nonlinearities. Appl. Math. Lett. 22(7), 1096-1101 (2009)

56. Xu, R., Meng, F.W.: Some new weakly singular integral inequalities and their applications to fractional differential equations. J. Inequal. Appl. 2016, Article ID 78 (2016)

57. Zhang, L.H., Zheng, Z.W.: Lyapunov type inequalities for the Riemann-Liouville fractional differential equations of higher order. Adv. Differ. Equ. 2017, Article ID 270 (2017)

58. Zheng, Z.W., Gao, X., Shao, J.: Some new generalized retarded inequalities for discontinuous functions and their applications. J. Inequal. Appl. 2016, Article ID 7 (2016)

59. Zhang, Q.M., Tang, X.H.: Lyapunov inequalities and stability for discrete linear Hamiltonian system. Appl. Math. Comput. 218, 574-582 (2011)

\section{Submit your manuscript to a SpringerOpen ${ }^{\odot}$ journal and benefit from:}

- Convenient online submission

- Rigorous peer review

- Open access: articles freely available online

- High visibility within the field

- Retaining the copyright to your article

Submit your next manuscript at $\boldsymbol{s p r i n g e r o p e n . c o m ~}$ 